\title{
Media Effects on High School and University Students' Views on Popular Clothes
}

\author{
Xiaomeng Ling ${ }^{1, *}, \dagger$, Liyu Yang ${ }^{2, \dagger}$, Chun Zhan ${ }^{3, \dagger}$, and Jingxin Zhao ${ }^{4, \dagger}$ \\ ${ }^{1}$ Chongqing NO.1 International Studies School, Chongqing, 401122, China \\ ${ }^{2}$ Shanghai Shixi High School, Shanghai, 200040, China, \\ ${ }^{3}$ Newcastle University, Media, Culture, Communication and cultural studies, School of Arts and Cultures, Newcastle \\ Upon Tyne, NE1 7RU, the Unite Kingdom \\ ${ }^{4}$ Beijing 21 st century international school, Beijing, 100142, China \\ *Corresponding author. Email: guanghua.ren@gecacademy.cn
}

${ }^{\dagger}$ These authors contributed equally.

\begin{abstract}
The theme of our research is the influence of popular logo on the behaviour of high school and college students. More and more high school and college students are attracted by popular brands, and it seems that popular logo becomes a fashion trend within those student communities. For this phenomenon, we consider that media plays an important role in this kind of consumer behaviour. In contemporary society, because of the rapid development of media, its influence needs to be taken seriously, especially on young people, which contributes to understand and change the consumer psychology of those people. In order to obtain relevant data for our research, we use a questionnaire with 15 individual questions. The answers to the questionnaire are used to analyse the correlation between the research question we proposed and the respondents' perspectives. Overall, we investigate respondents' opinions from three aspects, consumption idea, influence factor, and views on actions. In conclusion, the answers to the questionnaire and our analysis suggest that popular logos have an irreplaceable and unique domain in the clothing market nowadays. Meanwhile, social media greatly promote the popularity of those brands. It can be seen from the influence of external packing, spokesperson and indirect advertisement on these students' choices of popular brands. Besides, most young people pursuit those clothes mainly because popular logos fit their preferences and they are generally rational for the consume on popular logos. In the era of media, it is significant to realize the media effect and shape the correct consumption values of high school and college students on these popular brands.
\end{abstract}

Keywords: popular brands, social media, psychological comparison, imitative behavior

\section{INTRODUCTION}

In recent years, popular logo gradually occupies a dominant position in the clothing market. Popular logo has been popular for a few years. In English, popular logo means Streetwear. Streetwear is a casual style that began to gain worldwide acceptance in the 1990s. The core of popular logo is leisure and comfort. Its basic style is jeans, T-shirt, baseball cap and sneakers. Popular logo enthusiasts normally chase limited editions. Influenced by the surf culture of Los Angeles in the late '70s and early' 80s, popular logo became widely accepted. Ultimately, popular logo is defined as a multi-faceted, subculturally diverse, Southern California lifestyle "Tshirt" brand as opposed to a high-end luxury brand. Two elements are necessary for a street popular logo: T-shirts and uniqueness. Popular logo is the expression and manifestation of local culture. For example, we will go to the Forbidden City to find visual evidence that Chinese red can be used as a national fashion logo colour matching.

Li Ning is the most successful Chinese fashion brand in recent years, and it is also very popular with everyone. The reason why China Li Ning is more successful is that its popularity and public acceptance are higher than other fashion brands from other countries. In addition, another important element is that after its re-appearance in New York Fashion Week in 2018, the transformed China Li Ning has added more Chinese elements, starting from the 
Wutao series. Traditional Chinese elements such as Chinese embroidery, totem, mythical figures, myths and legends, landscape paintings are integrated into costume design, so that Chinese elements can be seen by Chinese people and people all over the world, and everyone can see this beauty. To carry forward the tradition, more people need to know the tradition, to develop the national tide, and more people need to support the national tide.

From the point of semiotics, popular logo has become a symbol. Eco has stated that semiotic is the study of signs, and their meanings in human social life [1]. This semiotic meaning has been used in marketing, such as unique aesthetic style and embedding the iconic logo in their products. As Kucuk argues that "brands can become inseparable semiotic entities, icons in a consumption environment" [2]. Meanwhile, popular brand also affects buyers' subconscious in their decisions. The semiotic of brand could promote the development of symbolic brand, every consumer could identify the brand through the simple logo, signs, and even colours [3]. Nowadays, it is a global trend that luxury fashion brands businesses to turn to social media to expand their marketing strategies. This is due to the nature of social media platforms, that is, the convenience provided by the Internet, which could allow users to communicate with each other and exchange information and opinions via different forms. Furthermore, a variety of social media platforms, such as Twitter and Facebook, have been widely adopted by luxury fashion industry and play a significant role in expanding the business [4].

With the popularity of street wears developed, we found that there are more people around us wear popular logo clothes. People can choose more kinds of popular logos than before. So we want to explore the views of contemporary high school and college students on the role of media. For the purposes to purchase popular logo clothes, some high school and college students like the design elements of popular logo. However, some students prefer to buy the popular logo clothes in order to fit in their surrounding social environment and imitate people's wearing styles around them. We think it is a kind of imitation behaviours and psychological comparisons. In our study, we focus on high school and college students' views and attitudes on popular logos. We used survey data to explore the influence of popular logo on the behaviour and psychology of high school and college students. The importance of our study is to investigate whether high school students and college students buy popular logo because of the value of popular logo itself, such as comfort and novel design, or because of the social phenomenon brought by popular logo, such as comparing behaviour, believing that buying expensive popular logo can improve their social status. The second part of the paper is the research method. The third is our survey data. We conducted the survey from four parts. The first is Basic information. We have a certain understanding of gender, age and their consumption amount in popular logo, so as to know whether their consumption level is in line with their age group. Secondly, Consumption Idea, we want to find out the reasons why they buy popular logo and the highest price they can accept. The goal is to find out how much they spend. Then there is the influence factor. We investigate what they think of popular logo, the reason for buying popular logo and the influence of popular logo on them. The aim is to understand what kind of thinking they are in when buying popular logo and what role media plays in the gap between popular logo and consumption. Then, Views on Actions. This is an open question, and finally, to understand the inner thoughts of consumers. Ultimately, conclusions and findings.

\section{RESEARCH METHOD}

We designed a questionnaire composed of 15 individual questions in order to collect data for our study. The questionnaire was given out online to the respondents in a WeChat program named Wenjuanxing. In total, there are 48 samples, of which 44 are valid. We divided the questionnaire into three parts, including consumption idea, influence factor and views on actions.

\section{RESULTS AND DISCUSSION}

\subsection{Preference of consumption}

There are 44 participants in this questionnaire survey. From figure 1, the number of female students is twice that of male students.

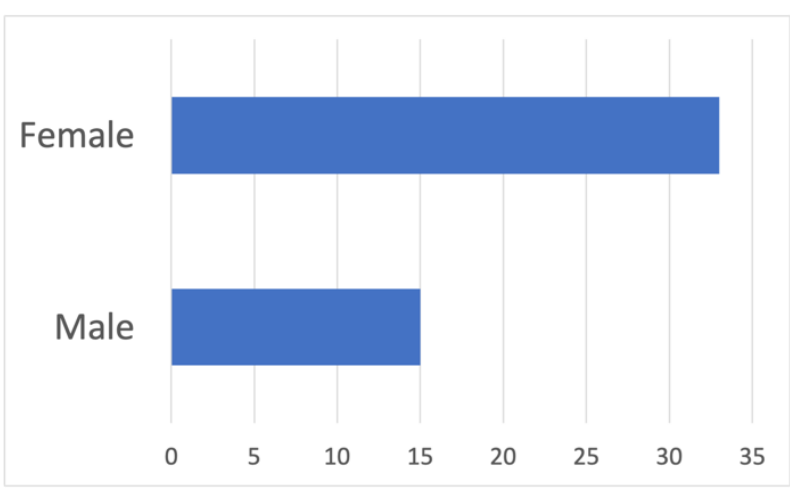

Figure 1 Gender of the respondent

From figure 2, we can know that people aged between 16 and 18 account for about half and those aged between 20 and 22 account for about one fifth. Therefore, the age of this sample is mainly young people aged between 16 and 22 . 


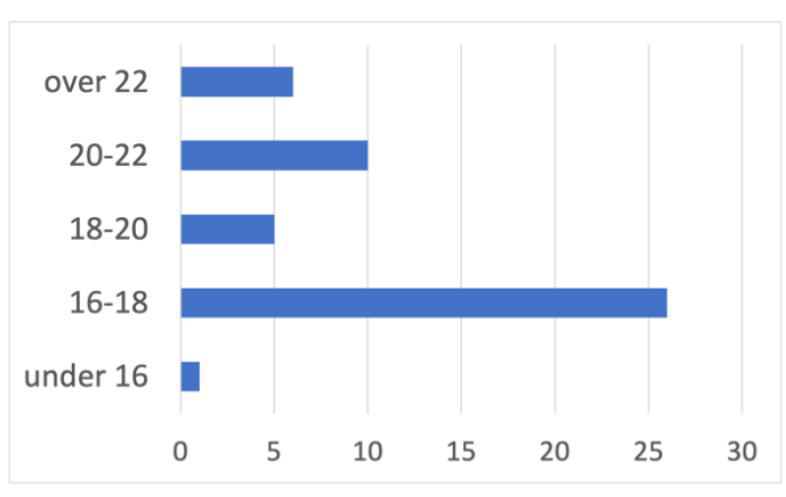

Figure 2 Age of the respondent

From figure 3, we can know more than $80 \%$ of them have experienced popular logo consumption. However, considering that young people aged 18-22 have the ability to make money, while people under 18 cannot for the time being, the consumption price of clothes purchased by them is investigated according to different ages, as well as the acceptable price range.

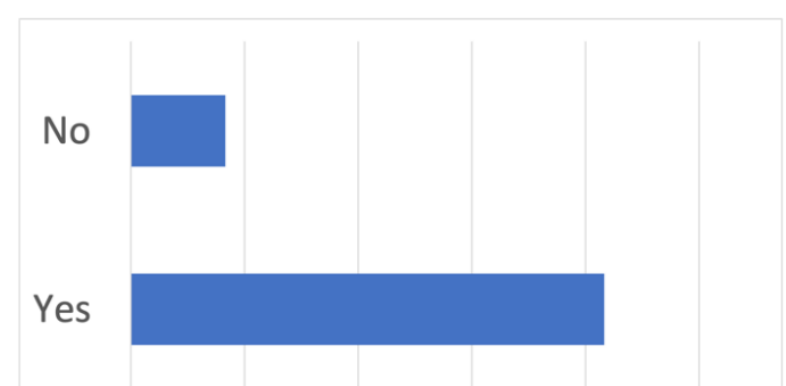

\section{$0.00 \% \quad 20.00 \% \quad 40.00 \% \quad 60.00 \% 80.00 \% 100.00 \%$}

Figure 3 The experience of buying popular clothes

Most of the participants' monthly popular logo consumption is less than 1000 yuan, accounting for about $60 \%$. The next is $1000-2000$ yuan, about $30 \%$. The consumption amount of 2000-3000 yuan and 3000 yuan accounts for the same proportion and is the least. At the same time, most of the people can accept the highest climax card price between 1000 and 1000-2000, they each account for about $40 \%$, among which a small number of people can accept the price of more than 2000. We suspect that such a result is related to income power, and people with income power can accept higher prices and consumption level. The vast majority of participants will give up consumption because of the high price of popular logo, and only about $12 \%$ of participants are willing to continue to spend. When people choose clothes, they will basically choose from the appearance, price, quality and style, and then take the brand and popularity into consideration. Among them, the appearance is design, which comes from the elements we like, and many elements and logos are formed by the influence of communication.
The results of the price choice are also reflected in the brand choice. Converse and Vans, which are priced at around 1,000 yuan or less, were purchased by more than half of the total number of participants, taking the first and second place, respectively. Supreme was second with half. Balenciaga's Rei Kawubo, Alexander Wang, Moschino and Off-White all account for more than 20\%, and their prices are around 2000-3000 yuan. It is worth noting that AMI, Miracle Kill and ASSC all have about $10 \%$ buyers, either because the price is too high or because the brand name is too low. These results are also in line with the results of the price survey.

Here are a few points about consumer preferences. The first is the consumption preference formed by consumption values. It reflects the influence of the cultural environment and cultural tradition on consumers. Different regions lead to the difference in their value evaluation and value orientation. Thus, it can be seen that the concept of consumption value is closely related to the traditional value and culture of each nation, followed by the consumption preference formed by the aesthetic orientation of consumption. The aesthetic orientation of consumption refers to people's appreciation and interest in the object of consumption. It is the result of the interaction between cultural environment and human psychology. Then there is the consumer preference shaped by the national disposition. Culture restricts people's character. First, it adjusts people's basic life attitude and basic concept, and second, it adjusts people's basic behaviour tendency. Finally, folklore leads to consumer preferences and taboos. Different countries and nations have their own unique and habitualized way of life, and have formed some traditional customs and habits that are different from other countries and nations in all aspects of life [5].

\subsection{Influence factors on popular logo}

From the result of high school and college students' consumption preferences, it is apparent that there are some factors influencing their chooses for popular brand. Therefore, it is necessary to analyse the reasons from other data.

According to data derived from figure 4 , it can be seen that there are about $80 \%$ of participants think the core of popular brand is a fashion trend, $72 \%$ of the total consider it is personalization and about half of people think it represents leisure and comfortable attitude. 


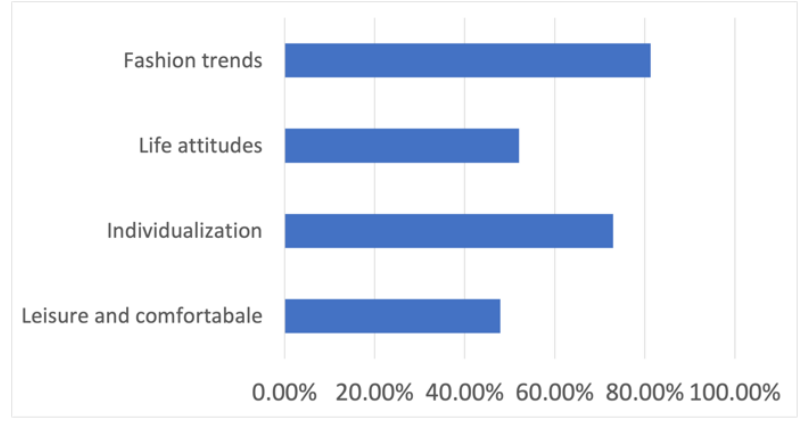

Figure 4 The core of popular brands

From Figure 5, there is approximately two thirds of people think the external packing of popular brands' products have certain influence on their chooses, a quarter of participants deem that the external packing has no affect for them, while there are no person believe the packing totally influence them, and few of people think the influence on them is great.

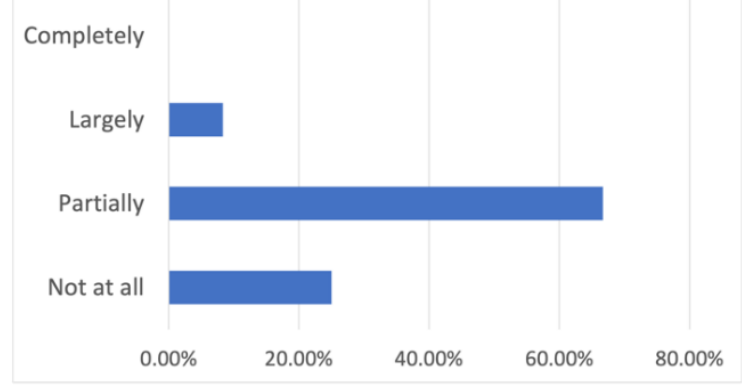

Figure 5 The extent of influence of packaging

Meanwhile, it can be seen from figure 6 that participants who believe the popular brand could give them confidence and good taste, only about $16 \%$ of the total amount think brand clothing bring status symbol, and a small part of people choose the "others"

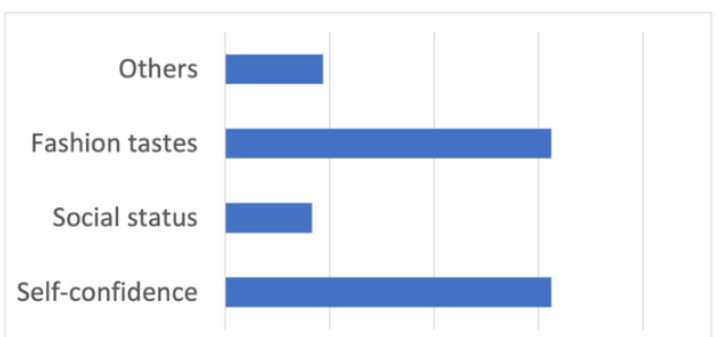

\section{$0.00 \% 20.00 \% 40.00 \% 60.00 \% 80.00 \%$}

Figure 6 Gain from buying popular clothing

In the aspect of whether the endorser affects the purchase behavior, it can be seen from figure 7 , participants who consider endorsers have influence on them occupied $60 \%$, while about $30 \%$ of the total do not think that endorsers have impact on them. Few people believe that endorsers have great affects, and only one participant deems that the consume behaviors are absolutely affected by celebrities.

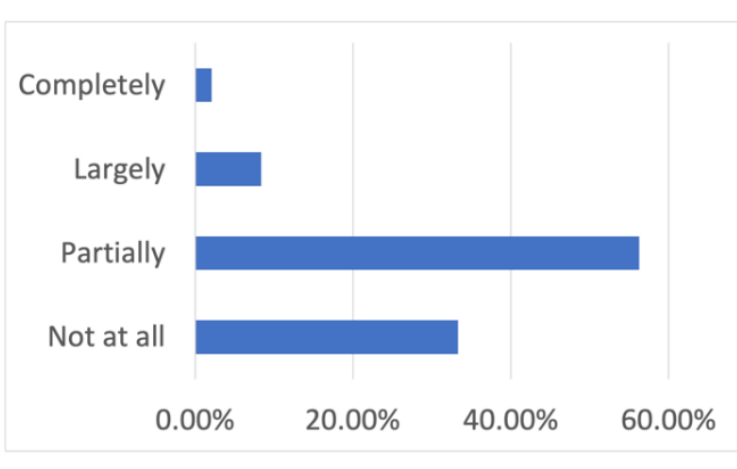

Figure 7 The extent of influence of spokesperson

From the result of the questionnaire, it is worth mentioning that the popular brand in the media has become a symbol, they have particular logos and styles, for example, when you look at sneakers with a star logo, you will think of Converse. Hence, it can be explained that popular brands may convert into popular symbols, enabling people to link the clothing of a certain brand to a specific representative symbol. As Holt argued that the symbolic brand has become the curtal symbols [6]. In brand marketing, semiotic is regarded as an important theory to construct the brand culture, from the aspect of semiotic psychology, consumers do not purchase products, they consume the meaning. Humans are a species driven by meaning and desire, they are makers of meaning, and express the meaning by creating and interpreting signs. It follows that people could unconsciously interpret things by associating them with a system of familiar conventions. Popular brands can use symbols to ensure the long-term effectiveness of brand messages in the consumer market [7]. In addition, the utilize of semiotics could improve the message inherent to the brand, convey the meaning that wants to create and affect consumers subconscious decisions. Those semiotic signs of brand usually presented by visual media, therefore, indirectly enhancing the influence of media on people's purchase behaviors on those brands.

Fashion magazines always reflect the popular trend, define the ideal beauty and affect the aesthetic of viewers. The collection that gives the design in the magazine more is to show the effect give color dazzle, which leads to targeted audiences notice those products and develop the idea that wants to them. Furthermore, films and TV series also influence people's preferences for popular brand. The creative combination of actors and actresses on screen is always charming, causing fans and audiences to intimate their outfits, particularly high school and college students because they are more likely to watch trendy drama, which indirectly affects their consumption behavior in popular brands.

Additionally, social media users can obtain the latest trends through online platforms and browse other people's shopping sharing and experience, which directly and indirectly attract viewers' shopping desires. In addition, advertising is also a key means of media 
influence. The unique videos and posters aesthetic style of popular logo clothing also attract the attention of consumers. The popular marketing team promotes their products through social media platforms, and the PR team works with the influencers on those platforms. The fans of these celebrities and micro-celebrities are attracted by their posters, which contribute to expanding the consumer base and increasing brand popularity. Besides, the design department captures the latest fashion trends and consumer suggestions through social media platforms, improving the design of products for catering to the needs of consumers. It can be said that the establishment of popular brands' official accounts on mainstream social media platforms has kept and enhanced the contact with consumers and improved the reputation of the brand.

High school and college students have become the major users of the media, there is no doubt that media has played an important role in the consumer behaviours of them, especially the popular brands.

\subsection{Views on buying popular clothes}

At the end of the questionnaire, we give two free response questions to ask about the respondents' own opinions on buying popular clothes from two aspects, the behavior of buying popular brands clothes and the comparison psychology in this respectively. We intend to further discover the psychological reasons behind this kind of consumption behavior.

The respondents were asked about their views on the behavior of purchasing clothes of popular brands, in particular, whether or not they think it is reasonable, healthy and whether it should be encouraged. To be more specific, this question shows what the role of buying popular clothes is in their daily life and how they think of it from a personal angle. Most of them think that the consumption of those clothes is understandable, but people should be rational and moderate, instead of blindly pursue it. Some people also suggest that they should form their own aesthetic taste and dress comfortably. Few think that buying popular brands clothes is completely unacceptable. We can see that the respondents are relatively subjective when answering the question, and most respondents consider buying luxury brands as a choice for individuals. Furthermore, it is people's own willingness to purchase any clothes they like. Buying popular brands clothes is merely one of the options that people could choose from according to their purchasing power and tastes in fashion. In the knowledge that a large proportion of the respondents have had bought clothes from those brands, the popularity of these fashion logos has certainly influenced their preferences when choosing their personal outfits by varying degrees. Also, research has shown that specialized characteristics labeled on products have strongly emphasized brand identity, which thus affects consumers' decisions on which brands exactly to buy [8].

In the next question, we introduced the idea of comparison psychology, as it is an important factor that contributes to the popularity of popular logos. Competition of wearing embodied in the value and popularity of brands of clothes people wear has always been discouraged in the society, and we think that there is a correlation between comparison psychology and increasing popularity of these brands. The respondents mentioned how they judge this sort of social comparison from various aspects. Regarding respondents' opinions on comparison psychology when consuming popular clothes, most people think that they should not buy trendy brands because of competition between their peers. Respondents assume that they should make decisions on clothing regarding their own preferences and consumption ability, and this kind of competition is not supposed to be advocated. Recent study shows that social comparison is one of the major factors that have great influence on Chinese consumers' attitudes towards purchasing luxury fashion goods [9]. And as for contemporary teenagers and young adults, they, in particular, are in a social atmosphere where materialism is prioritized by varying degrees. Although social comparison in wealth showed by people's wearing is universally regarded as an example of vanity and superficialness, wearing clothes with the most popular brand logo on is, indeed, still a trend. According to Leon Festinger's social comparison theory, individuals tend to compare their appearance to others to assess their own perceptions and abilities [10].

Thus, one of the underlying causes of this phenomenon may be that some people believe that wearing clothes with popular logo is able to socially accepted and welcomed by their peers and they wear those logos to express the idea that they want to fit in the modern social group of the young generation, because they demonstrate their attitudes towards fashion and financial status through those logos as well as the price level behind.

\section{CONCLUSION}

With the number of popular brands increases, people can choose many kinds of different popular brands. Unique styles attract young people to buy popular logo clothes. Local culture is reflected in popular logos, and the company of popular logo designs some creative and aesthetic clothes under the background, that is the reason that some 'Guochao' brands get many young people good graces. Popular brands also affect consumers' decisions from the perspective of semiotics, and social media provides the popular logo a platform to popularize their awareness. More and more high school and college students choose to purchase the popular logo clothes, we collect data to explore the main reason to urge high 
school and college students to buy them. And we think it is an imitation behaviors and psychological comparison among them.

We use a questionnaire that contains 15 individual questions. We got 48 samples in total, and 4 of them are null, so there are 44 effective questionnaires. Our main objects are high school and college students, so most of our questionnaire objects are 16-22 years old. Through the questionnaire data, we clearly know some basic information about high school and college students. And we use these data to analyse the certain reason why high school and college students want to buy popular logo clothes.

From the consequences, we found that all the popular brands have their unique style, and this makes people can recognize them quickly, and social media help the brand a lot for increasing the popularities of popular brands.

The phenomenon of this research reflects some results, more than half of the high school and college students do not purchase overpriced popular logo clothes. The sequence of choosing is appearance, price, quality, style and the popularity of this brand. And there are most people think the core of popular logo is fashion, and then it is the comfortable type. These results clearly present those students' preferences and attitudes for the consumption of popular brands.

These results also show some influence factors. The imitation behavior and psychological comparison affect young people to purchase the popular logo clothes. The people think popular logo can let them be more confident in their situation, and they also think wearing popular logo can increase their taste and station, but not the real comfortable feeling and the designing idea. Additionally, social media has played an important role in influencing the consumer behaviors of high school and college students.

They could obtain information about fashion trend from those platforms and be attracted by various advertisements. Actually, social media express a correct value to the young people who are still in high schools or colleges. They need to adjust their attitudes in a right way to recognize the popular brand, and do not purchase these clothes in order to compare with others. The popular brand has its own unique value, people should understand them in a correct way.

\section{REFERENCES}

[1] Eco, U. (1976). A Theory of Semiotics. Bloomington: Indiana University Press.

[2] Kucus, S. (2014) A semiotic analysis of consumergenerated antibranding. Marketing Theory, 15(2), 243.
[3] Kucus, S. (2014) A semiotic analysis of consumergenerated antibranding. Marketing Theory, 15(2), 243.

[4] Angella Jiyoung Kim, Eunju Ko, Journal of Global Fashion Marketing 1 (3), 164-171, 2010

[5] Wenku.baidu.com. (2021). The literature review of consumer behaviors - Baidu library. [online] Retrieved May, 27, 2021,from <https://wenku.baidu.com/view/2094a1316e1aff00 bed5b9f3f90f76c660374c21.html>

[6] Douglas, H. (2005). How Societies Desire Brands: Using Cultural Theory to Explain Brand Symbolism. In Inside Consumption: Consumer Motives, Goals, and Desires, edited by S. Rathneshwar, David G. Mick and Cynthia H, 292 308. London: Routledge.

[7] Rossolatos, G. (2017). Brand image re-revisited: a semiotic note on brand iconicity and brand symbols. Social Semiotics, 28(3), 412-428.

[8] Angella Ji-Young Kim, Eun-Ju Ko, The impact of design characteristics on brand attitude and purchase intention-focus on luxury fashion brands, Journal of the Korean Society of Clothing and Textiles 34 (2), 252-265, 2010)

[9] Bopeng Zhang, Jung-Kwan Kim, Luxury fashion consumption in China: Factors affecting attitude and purchase intent, Journal of Retailing and Consumer Services, Volume 20, Issue 1, 2013

[10] Festinger, L. (1954). A theory of social comparison processes. Human Relations, 7(2) 117-140 